\title{
Z-Degrees: Four Colleges in 12 Months
}

\author{
Timothy R. Anderson \\ Educational Innovations \\ Stephen M. Kelly \\ Educational Innovations \\ Kim Lynch
Educational Innovations
}

Educational Innovations at the Minnesota State system office assisted four colleges in creating Z-degrees in just 12 months. This work was made possible through a $\$ 500,000$ allocation from the Minnesota state legislature. Structures and strategies were developed to assess campus readiness, apply tools and support, and provide leadership throughout implementation. Attention was also given to reporting mechanisms and program sustainability with an eye toward future implementations of new and expansion of existing Zdegree programs. From these experiences, recommendations emerge to help inform other systems considering Z-degree initiatives.

Keywords: OER, Open Educational Resources, Z-degree

\section{INTRODUCTION}

At this moment, there is a student at your college or university who is ready to drop out of a course. It is not because their instructor does not know their name. It is not because the coursework is too challenging or involves too much effort. It is not even because their personal life is disrupting their studies in an untenable way.

This student is among the roughly $23 \%$ of all students who cannot afford the course textbook (Florida Virtual Campus, 2018).

Put another way, if your campus had 1000 full-time students, approximately 230 might fail to complete a course because they cannot afford the associated textbook.

What if there was a way to prevent this - would you pursue it? This article is about our efforts to meet this challenge through the creation of Z-degrees. Z-degrees are particularly effective at eliminating the cost of expensive publisher textbooks and allowing equitable access to courses. When executed effectively, they can foster a culture of inclusion and diversify teaching and learning. We share some of the structures we created and strategies we applied, and along the way, we share some provisional outcomes and recommendations. We hope that our story will entice other system leaders to action, believing they too can address this troublesome financial barrier confronting students and eliminate dropout rates due to an otherwise manageable antagonist - the expensive publisher textbook. 
Minnesota State Colleges and Universities (Minnesota State) is the third-largest system of state colleges and universities in the United States with 30 state community and technical colleges, seven universities, and comprising 54 campuses. Minnesota State serves 340,000+ students each year, with more students of color and American Indian students attending the colleges and universities of Minnesota State than all other higher education providers in Minnesota combined.

Educational Innovations is a unit of Academic and Student Affairs at the Minnesota State system office. Our unit is an energetic team that seeks to identify, seed, and support innovative technologies and talent to drive and enable the highest-quality teaching and learning experiences for students across Minnesota State. Z-degrees and Open Educational Resources (OER) are system priorities of the Educational Innovations unit.

\section{WHY A Z-DEGREE?}

Systems and higher education institutions may choose to create Z-degrees for various financial and programmatic reasons. Still, the positive financial impact a Z-degree has on student success is typically most prominent. Consider some factors we as educators have learned in just the past decade of inquiry on textbook affordability:

- The average student spends $\$ 1,200$ each year on textbooks and course materials (Bliss, 2015).

- $\$ 1,200$ per year is roughly $40 \%$ of tuition at a two-year community college and $13 \%$ of tuition at a four-year public university (Senack, 2015). In Minnesota State, some students have begun referring to textbooks as their "second tuition."

- In some circumstances, the cost of textbooks and course materials exceeds tuition costs (Bliss, 2015; Fischer, et al., 2015).

- Research suggests $65 \%$ of students may not purchase a textbook because of cost. Furthermore, one-half of those students report significant concerns about either failing a course or limiting their course load due to their difficulty acquiring a textbook (U.S. PIRG, 2014).

A Z-degree with high-quality OER reduces textbook cost, promotes equitable day-one access to course materials, encourages increased course loads, and supports pedagogical creativity among faculty. These conditions feed student success. One Georgia study found that OER improves grades and decreases the occurrence of D, F, and Withdrawal letter grades; moreover, the study indicated a higher rate of letter grade improvement for Pell, part-time, and underserved students in higher education (Colvard, Watson, \& Park, 2018). A study from Fischer, et al. (2015) suggests students who enroll in an OER course one semester are more likely to increase their credit load the following semester.

These data are representative of the broad U.S. student population. Still, a deeper dive into textbook affordability studies reveals that more dire circumstances confront our financially disadvantaged students and students of color. As Griffiths, et al. (2020) suggest, low-income and underrepresented minority students are disproportionately impacted by textbook costs, implying that textbook cost reduction would support equity goals. During the early $2000 \mathrm{~s}$, as many as 1.4 to 2.4 million students from low to moderateincome families were expected to not attain a bachelor's degree due to financial barriers (Advisory Committee on Student Financial Assistance, 2006). Considering the cost of textbooks has increased 1,041\% over the last 40 years (Popken, 2015), it is fair to implicate textbook cost in this failure of U.S. higher education.

Z-degrees serve all students well, but Minnesota State Z-degrees help our most financially disadvantaged students. We think it likely a Z-degree would be similarly effective at institutions outside of our system 


\section{IMPLEMENTING A Z-DEGREE AT A SYSTEM}

\section{Define Z-Degree and Zero-Textbook-Cost Courses}

Our work in developing Z-degrees suggests that many higher education colleagues are still unfamiliar with the Z-degree concept and how it is defined. We strongly recommend establishing a definition early. For example, we define a Z-degree as a two or four-year degree program that exclusively uses zerotextbook-cost (ZTC) materials such as open educational resources (OER) and open textbooks. Regardless of your definition, a clear definition is essential to ensure consistent application and accurate implementation across courses.

To further elaborate on our definition, an instructor in one of our Z-Degree programs must ensure materials in the left column are available in a digital format at no cost. In contrast, students may incur costs related to the materials in the right column.

TABLE 1

RESOURCES ALLOWED AND EXEMPTED FROM Z-DEGREES

\begin{tabular}{|c|c|}
\hline ZTC Materials & Standard Cost Materials Not Included in ZTC \\
\hline 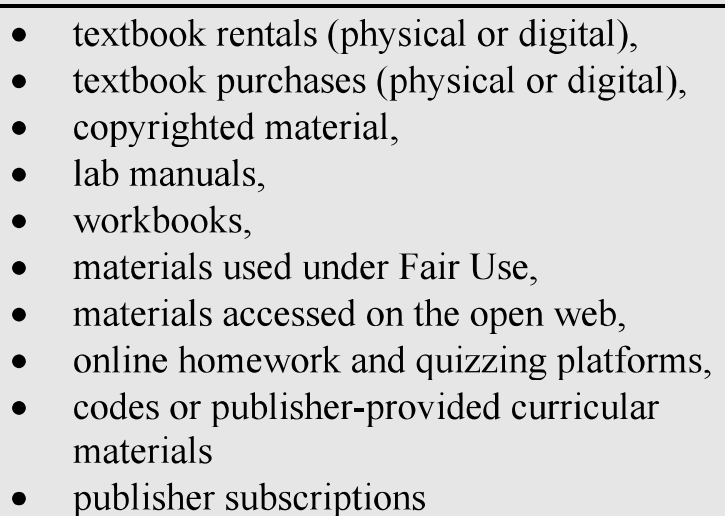 & $\begin{array}{l}\text { - } \text { art supplies, } \\
\text { - } \text { calculators, } \\
\text { - } \text { equipment, } \\
\text { - } \quad \text { Requiss fees: fees not included: } \\
\text { be charged by all colleges and universities. } \\
\text { - Campus discretionary fees established by the } \\
\text { board and adopted by the college. } \\
\text { - Personal property, service charges or course } \\
\text { activities having value outside of the } \\
\text { classroom }\end{array}$ \\
\hline
\end{tabular}

\section{Assessing Campus Readiness}

Creating a Z-degree is challenging and becomes even more challenging when aggressive time constraints are imposed. Based on our experience working directly with system colleges and universities, we compiled a list of criteria against which we could assess campus Z-degree readiness.

We invited all 30 colleges within Minnesota State to apply for our Z-degree program. College applicants completed an interest application and readiness assessment to measure their preparedness to pursue a Z-degree. This readiness assessment collected responses to the five following categories:

- Readiness of campus personnel: The current level of individual and collaborative engagement in OER and textbook affordability initiatives, specifically focusing on campus administrators, faculty, librarians, instructional designers, instructional technologies, and others the campus personnel identified as relevant to its local efforts.

- Structural readiness: The formal groups and roles an institution recognizes that directly serve the OER or no-cost needs of faculty and students, including OER committees, designated OER librarians or staff, campus bookstore collaborations, formalized strategies around OER development and sustainability, and organized textbook affordability initiatives.

- Cultural readiness: The less formal or incidental activities that foster awareness and appreciation for OER and no-cost materials, including campus-wide conversations around textbook affordability, OER advocacy among students and student senates, OER workshops for faculty and staff, and mentoring programs for faculty interested in engaging OER. 
- Current open and zero-textbook-cost inventory: A listing of courses and faculty already using OER or no-cost course materials. (This information is beneficial in assessing distance to a Z-degree finish line)

- Proposed z-degree and course sequence: A listing of courses in sequence comprising the proposed Z-degree. (In our case, we applied preference to proposals fulfilling transfer pathway requirements or focusing on high-demand career fields)

The college responses to this assessment suggested that colleges were at different levels of preparedness. We awarded funding to the six colleges that fulfilled the greatest number of the above criteria.

\section{Building an OER Foundation to Support Z-Degree Readiness}

Building OER awareness, skills, and capabilities in Minnesota State is a six-year running system priority. Educational Innovations offers activities that provide crucial support for campus Z-degree and OER readiness. Examples of these activities include system-wide events and workshops on OER and textbook affordability, project support and guidance, technical support, and seed funding for campus-based initiatives.

\section{Develop an OER Steering Committee}

Preparing a Z-degree course can be complicated and time-intensive since many faculty members are deeply committed to materials and technology they have used for years or even decades. Faculty are less likely to convert their courses unless they are aware of OER in their discipline and have confidence in the open textbooks' quality (Hilton, 2016). Beile, deNoyelles, and Raible (2020) recognized that some faculty still have the perception "you get what you pay for" with regard to OER, and this sentiment is prevalent today despite many studies that demonstrate the quality of OER is often equal to or better than publisher texts (Hilton 2016; VanderWaal and Gucinski 2019).

To help overcome these hurdles and usher the work forward, multiple institutions created a faculty steering committee consisting of faculty, administrators, librarians, and OER subject matter experts who assisted with intellectual property and content licensing questions.

Institutions included existing faculty on their OER steering committee. These faculty served as important ambassadors to other faculty and entire departments, helping determine general interest among colleagues, offering support for campus workshops, and extending one-on-one time through faculty office hours.

Z-degree institutions also indicated that a steering committee provided a broader cultural benefit, acting as a clearinghouse for questions related to myths, misinformation, and reasonable concerns surrounding Zdegrees and OER. Some of these questions included:

- Are OER and open textbooks high quality?

- How will a Z-degree impact my academic freedom?

- Will this remove a faculty member's ability to author and profit from a textbook?

- How can our campus manage faculty turnover in ZTC courses?

- Will ZTC courses be cut due to low enrollment?

\section{Events and Workshops}

OER learning circles. Educational Innovations sponsors OER learning circles that guide faculty in creating, adopting, and adapting OER. These learning circles also act as collaboration incubators that can spin-off projects among disciplinary faculty from different campuses in the system. These circles have helped foster a system-wide community of OER practitioners, and they are a proven model for supporting parallel Z-degree development on multiple campuses.

Through these learning circles, faculty share their challenges and successes, and they provide each other mutual support with only a short time to complete. Along the way, faculty address accessibility, equity and diversity, align their materials to course objectives, and tailor instructional materials to address each learner's inclusive needs. 
One hundred thirty-eight faculty across Minnesota State participated in a system sponsored OER learning circle from $2018-2020$.

OER Librarian's Workshop. Annual events such as the OER librarian's workshop day allow participants to:

- Gain awareness of OER projects and activities system-wide

- Build a community of librarians to support faculty in OER

- Acquire tools and information to increase comfort with creative commons licensing and copyright issues for OER

- Gain awareness of opportunities in innovation funding for OER

- Articulate and create action plans for engagement across campuses

The workshops held in 2018, 2019, and 2020 had 155 total attendees, with attendance increasing each year.

\section{OER Community Conversations}

OER Community Conversations are open-forum events for all to share, discuss, and learn from colleagues in the Minnesota State OER community. Hosted by the OER Faculty Development Coordinator, OER Community Conversations are virtual, hour-long meetings held twice per semester and often feature a guest speaker on an OER topic of interest.

\section{Creative Commons Certificate}

Creative Commons (CC) certification is the result of an in-depth course covering CC licenses, open practices, and the Creative Commons' ethos. Participants engage in readings, quizzes, discussions, and practical exercises to develop open skills. The CC team offers personalized engagement with expert facilitators and copyright lawyers in the field. The CC certificate powers participants to better advise their institution on creating and engaging with openly licensed works. Participants also learn how to adapt and innovate on existing openly licensed materials (Creative Commons, 2020).

Minnesota State supports educators and librarians financially to obtain the Creative Commons (CC) certificate. When Minnesota State first engaged in funding CC certificates, the goal was to have an expert educator and or librarian in CC licensing at each Minnesota State institution. To date, 17 educators and 25 librarians completed the certificate programs and are now $\mathrm{CC}$ licensing experts for their campuses and have the skills to advise their institutions on creating and engaging with openly licensed works.

Building Community around Textbook Affordability and OER. Minnesota State's commitment to the textbook affordability community allows OER professionals to share common interests in developing highquality zero-textbook-cost (ZTC) courses. SharePoint sites provide access to many sources to create, adopt, and adapt OER. The community also allows for collaboration on best practices and enables members to solicit advice from like-minded professionals.

Within the community site, we offer many resources to faculty to convert courses to ZTC. Examples of these resources include:

- By discipline, quick-start guides allow community members to find ZTC resources to create, adapt, and adopt a ZTC course.

- Answers to frequently asked questions around Z-degree and OER

- Printing and publishing resources and information

- Glossary of terms

- Calendar for textbook affordability events

\section{Fund Z-Degree Development}

Many institutions find it challenging to fund Z-degree development in a time of declining enrollments and strapped budgets. These circumstances provide the perfect opportunity for both state government and system offices prepared to make investments that directly impact students' financial well-being. At 
Minnesota State, we view this as a moral imperative shared between our state legislature, Educational Innovations, and our statewide student associations.

\section{State Legislative Funding}

Minnesota State was fortunate to receive a one-time $\$ 500,000$ allocation from the Minnesota legislature to create Z-degrees at three system colleges in academic year 2020-2021. Educational Innovations combined these funds with a one-time matching allocation from the system, forming the resource base of the Z-degree program presented in this article.

\section{Minnesota State Funding}

There was strong interest in the Z-degree opportunity, so Minnesota State provided one-time matching funds to maximize the Z-degree efforts. The legislative funding and matching funds by the system office allowed for creating two types of grants. The Z-degree large grant awarded \$100,000 (large grant) to six colleges to fund and support Z-degree development and implementation. The funding also provided $\$ 10,000$ (small grant) to four colleges to maintain momentum toward future Z-degrees.

\section{Guidance for Campus Implementation of Z-Degrees}

Campuses will confront various challenges as they pursue implementation of their Z-degrees, and a system or central office should be prepared to provide both guidance and tools when needed. The top five actionable requirements allowed us to inject our experience and leadership in developing a successful Zdegree.

1. Attain faculty participation

2. Organize an OER steering committee

3. Ensure library support

4. Engage stakeholders

5. Reach students

\section{Attain Faculty Participation}

Each institution will experience some unique challenges when recruiting faculty to work on a Z-degree. Still, through a combination of tools and resources, a system can help address various circumstances. Our experience suggests campuses that score highly on a readiness assessment will experience less inertia as they launch their Z-degree initiative. The opposite is true of campuses that score poorly on readiness assessments. In short, campuses with a history of promoting OER and textbook affordability more easily create Z-degrees.

The campuses scoring higher readiness levels exhibited some of the most common structural and cultural features we encourage and look for. For instance, some had OER steering committees and a recognizable OER culture supported by faculty and librarian champions. These features made faculty recruitment profoundly easier, whereas campuses lacking these features tended to struggle more in convincing faculty to participate

All campuses recognized the importance of faculty compensation as a necessary recruitment tool, and although these were effective as initial incentives, money alone was inadequate, and faculty needed more support. We collaborated with campus leaders to provide a variety of professional development workshops delving into topics such as:

- Z-degree program requirements and expectations

- Copyright and intellectual property

- Accessibility and usability

- Course design

- Accessing no-cost materials or textbooks through libraries

- Resources for searching and locating OER content 
Colleges also self-developed programs to recognize faculty contributions, build esteem for Z-degree work, and encourage faculty hesitant to join the initiative. By way of example, Lake Superior College created a Zero-Hero program that recognizes faculty who convert their course for the Z-degree. These faculty receive a Z-pin to wear that signifies their accomplishment and acts as a small Z-degree promotional tool. This program alone created more participatory interest among faculty than the college could accommodate!

\section{Ensure Library Support}

There is an increasingly robust volume of OER available on the web, and faculty are often left sifting through seemingly endless lists of sites containing OER content. Librarians can lend their expertise and help faculty conduct more efficient OER searches through one-on-one training and guidance. Librarians at Z-degree campuses have also created lib-guides to help faculty searching for materials appropriate for their Z-degree course.

We cannot emphasize enough the value librarians bring to system OER initiatives and campus Z-degree initiatives. Our annual OER Librarian Workshop routinely reaches maximum capacity, indicating the sustained commitment librarians make on system campuses. Librarians are the primary support for system sponsored OER Behind the Scenes workshops where attendees examine OER development topics, such as choosing an OER platform, system and campus OER processes, and necessary forms that support OER creation.

\section{Engage Stakeholders}

Identifying and acknowledging stakeholder groups early in the process is critical to Z-degree success. In our case, we recognized the importance of inviting faculty collective bargaining units into the project discussions from the beginning. We emphasized the union representing our two-year college faculty since the Z-degree work would occur on our two-year campuses. We intended to ensure processes that conformed to the obligations established in applicable contracts created to ensure successful Z-degree outcomes.

Faculty representatives helped unearth serious concerns among faculty. For instance, the bargaining agreements provide broad faculty discretion in selecting course materials. Some faculty perceived that converting their course to OER was limiting that freedom rather than expanding it. Campus administrators engaging leadership early in the process avoided potential Z-degree roadblocks, such as a faculty member in a department-of-one who might refuse to convert a course. Hence, they could put the stop on Z-degree.

\section{Reach Students}

No innovation has much value to students if they do not know it exists, and a Z-degree is no exception. While the colleges allocated portions of their budgets to student outreach, one Z-degree college nearly spent their entire award on promotional efforts. Five of the six colleges created web pages dedicated to their Zdegree where students could learn more about the Z-degree and associated courses. These sites provide general Z-degree and OER FAQs, instructions for Z-degree course registration, campus contacts, and campus commitments to lower education costs through textbook affordability programs.

\section{PROJECT MANAGEMENT - MOVING FORWARD}

A project manager from the system office was assigned to help facilitate and track campus progress toward Z-degree development. This project manager helped identify budgets, communicate Z-degree progress, and develop a collaborative workspace and resource site.

Minnesota State was allowed just one year to develop at least three Z-degrees, so the project manager used a standardized tracking spreadsheet as an evaluation plan to ensure course development was within that year. The evaluation plan tracked the status of all courses identified for the campus Z-degree. It allowed the campus administration to track the status of each course to ensure full Z-degree compliance. 
When appropriate, the project manager offered additional resources to those campuses that may not be on track for meeting Z-degree deadlines. Examples of system office resources included an intellectual property attorney, faculty development resources, accessibility, information technology, and research.

\section{Measuring Z-Degree Success Reporting}

During the development of Z-degrees, the project manager required campuses to update their Z-degree tracking spreadsheets monthly. These updates were used to report project status and projected cost savings per college. The reporting included:

- Conversion status (e.g., not converted, converted, future plans to convert, etc.)

- On-track status (semester and year expected for completion)

- Number of sections per course converting to ZTC

The report serves two purposes, (1) provide transparency to faculty, students, and Minnesota State legislators on campus progress, and (2) motivate faculty who teach or are planning to instruct ZTC course by displaying total projected cost savings to students. We aggregated this information to create a report that we made available on Minnstate.edu, see figure 1.

FIGURE 1

\section{Z-DEGREE PROGRESS REPORT}

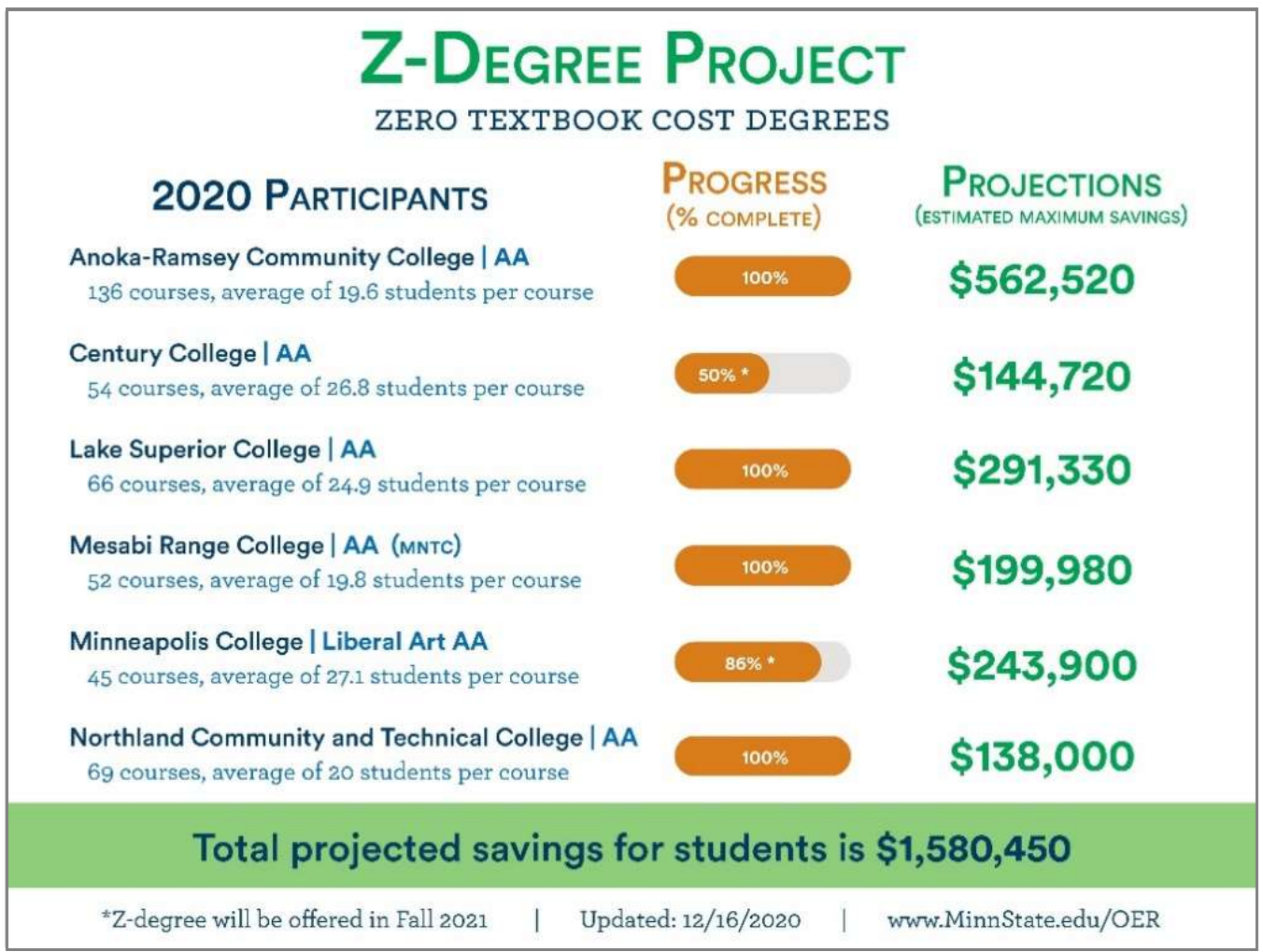

\section{SUSTAINABILITY AND THE FUTURE}

Minnesota State successfully developed four Z-degrees in one year, with another two colleges completing by Fall 2021. To continue this momentum and create additional Z-degree offerings, the Minnesota State system office invited other colleges to submit an interest application to receive fiscal year 2021 funding (Z-degree FY21) for one of three grant types: 
1. Explore - Prepare a foundation for Z-degree development. Activities may include:

- Faculty development

- Open textbook exploration and review

- Building open textbook capacity in the transfer curriculum

- Funding up to $\$ 25,000$

2. Implement - Create, finalize, and implement a new Z-degree. Activities may include:

- Course development for Z-degree with at least two distinct courses in each transfer curriculum goal area and at least enough credits in each transfer curriculum goal area to complete the transfer curriculum package

- Ability to offer a Z-degree for Fall of 2022

- Funding up to $\$ 100,000$

3. Expand - Increase the number and type of courses offered in an existing Z-degree. Activities may include:

- Add courses or course sections

- Increase the number of faculty using zero textbook cost materials in a course already part of an existing Z-degree

- Funding up to $\$ 25,000$

\section{RECOMMENDATIONS}

As a result of our ambitious goal of creating Z-degrees in one year, we learned a great deal. Our experience allows us to provide the following recommendations:

- Research Z-degrees, OER, and other ZTC related subjects. Campuses should conduct this research before taking on a Z-degree effort. We were not the first system to take on Z-degrees. In the spirit of openness, many consortia, systems, and colleges have shared their experiences with implementing full Z-degrees, including best practices, toolkits, and the benefits of ZTC in increasing college access and success. This information can be invaluable and can even allow for the replication of successful Z-degree implementations.

- Define the terminology associated with the development of Z-degree courses. For example, according to Wiley (2016), "open" has caused considerable confusion in the current education innovation discourse. When developing Z-degrees, we often use the term "open," referring to textbooks and course materials within Z-degree courses. Terms include open education resources, open-access, open pedagogy, open, repository, open textbooks, etc.

- Invite labor organizations to the Z-degree discussions. We had heard from faculty and labor organizations that too much focus is on cost savings, and there are other reasons why faculty choose textbooks. While our research has demonstrated significant student success in the use of OER, we could have been more deliberate in linking Z-degrees to student equity and guided pathways (California Community Colleges, 2018).

- Create a sustainability plan. While many faculty are willing to convert their course to a ZTC course, we have heard concerns about those courses' sustainability. Minnesota State is addressing these concerns by ensuring an infrastructure exists that allows faculty to locate OER, offer opportunities to have OER reviewed, and support open licensing questions.

- Provide appropriate technologies. Z-degrees require faculty to move away from publishersupported platforms. Ensure technologies exist to support the storage and management of ZTC resources.

- Invite bookstore representation. It is essential to understand bookstore governance and how they can and cannot support Z-degree resources. For those bookstores where governance allows, they can plan a critical role in Z-degree initiatives, including:

- Maintain textbooks lists, including those that are zero-cost 
- Can offer valuable information in campus- or system-wide OER and Z-degree initiatives

- Offer on-demand printing services for OER textbooks

\section{CONCLUSION}

This article revealed the structures, strategies, and tools we employed to launch four Z-degrees in 12 months within one of the largest public higher education systems in the United States.

We made the financial case for Z-degrees through a brief review of the literature. This review demonstrates, among other things, the high cost of course materials, the financial barrier to access, the rapid inflation of textbook costs, and the increasingly held belief that textbooks represent a form of the second tuition. We further suggested the Z-degree's potential ability to level student success opportunities, particularly for Pell, part-time, and underserved students in higher education. A Z-degree strongly impacts our most financially disadvantaged students.

We emphasized the importance of definitions to ensure consistent application and accurate implementation across courses. The definitions should be a first order of business for all system and campus leaders planning to launch a Z-degree.

Among the many structures and strategies, we shared, a readiness assessment embedded within an interest application proved beneficial. We recommend system leaders ground their efforts by identifying those criteria that position campuses for success and then encourage campuses to align their priorities to those criteria.

We further underscored the benefits of building a culture of OER and textbook affordability even before launching a system or campus-based Z-degree initiative. Some of the more common tools and practices we observe campuses employing include getting all the right campus personnel and students involved, engaging the campus community in intentional conversations, and emphasizing faculty development through offerings like learning circles and OER certifications.

While acknowledging the unique challenges campuses may confront, we offered guidance in five areas of practice common to all our campus Z-degree efforts. This guidance touched upon attaining faculty participation, establishing an OER steering committee, ensuring library support, engaging stakeholders, and effectively reaching students.

We noted the importance of both strong project management and a well-drawn program for reporting. While we continue looking at Z-degree sustainability, we admit this appears not as straightforward as we would prefer. Our current sustainability approach includes the continued maintenance of an online repository for Z-degree materials and a continued commitment to funding opportunities that can seed and expand Z-degree initiatives within the system. We are eager to learn more about sustainability from others working in this space.

Our experience suggests system and campus leaders should take a cue from our students and do their homework. The body of literature and expertise on Z-degrees is growing and awaits researchers. Many professional organizations like the Open Education Network filled with knowledgeable practitioners prepared to share their experience in the true spirit of open. Experts share their insights, Z-degree models, and OER development in communities like these.

We further advise system and campus leaders to stay apprised of the changing opinions and issues surrounding Z-degrees and the open movement. This work has historically focused on easing the financial burden of textbooks costs. Still, increasingly the emphasis is shifting to concentrate on its positive impacts on teaching, learning, and student success. This shift resonates with many faculty members, and in retrospect, we should have more deliberately linked Z-degrees to student equity and guided pathways (California Community Colleges, 2018). Realizations like this provide an impetus for all the excellent work that lies ahead. 


\section{REFERENCES}

Advisory Committee on Student Financial Assistance. (2006). Mortgaging our future: How financial barriers to college undercut America's global competitiveness. Washington, DC: Advisory Committee on Student Financial Assistance. Retrieved from https://files.eric.ed.gov/fulltext/ED529499.pdf

Beile, P., deNoyelles, A., \& Raible, J. (2020). Analysis of an open textbook adoption in an American history course: Impact on student academic outcomes and behaviors. College \& Research Libraries, 81(4), 721-736. doi: 10.5860/crl.81.4.721

Bliss, T. (2015). Z as in zero: Increasing college access and success through zero-textbook-cost degrees. William + Flora Hewlett Foundation. Retrieved from https://hewlett.org/z-as-in-zeroincreasing-college-access-and-success-through-zero-textbook-cost-degrees/

California Community Colleges. (2018). Implementing California's Zero-Textbook-Cost Degree Program. Retrieved from http://onlineteachingconference.org/wpcontent/uploads/2018/06/DALY-Implementing-Californias-Zero.pdf

Colvard, N.B., Watson, C.E., \& Park, H. (2018). The impact of open educational resources on various student success metrics. International Journal of Teaching and Learning in Higher Education, 30(2), 262-276. Retrieved from https://eric.ed.gov/contentdelivery/servlet/ERICServlet?accno=EJ1184998

Creative Commons. (n.d.). Creative Commons Certificate. Retrieved from https://certificates.creativecommons.org

Fischer, L., Hilton, J., Robinson, T.J., \& Wiley, D. (2015). A multi-institutional study of the impact of open textbook adoption on the learning outcomes of post-secondary students. Journal of Computing in Higher Education, 27, 159-172. doi: 10.1007/s12528-015-9101-x

Florida Virtual Campus. (2018). 2018 student textbook and course materials survey. Retrieved from https://dlss.flvc.org/documents/210036/1314923/2018+Student+Textbook+and+Course+Material s+Survey+-+Executive+Summary.pdf/3c0970b0-ea4b-9407-7119-0477f7290a8b

Griffiths, R., Mislevy, J., Wang, S., Ball, A., Shear, L., \& Desrochers, D. (2020). OER at scale: The academic and economic outcomes of Achieving the Dream's OER degree initiative. Menlo Park, CA: SRI International.

Hilton, J. (2016). Open educational resources and college textbook choices: a review of research on efficacy and perceptions. Educational Technology Research \& Development, 64, 573-590. doi: 10.1007/s11423-016-9434-9

Minnesota State Colleges and Universities. (2019). Minnesota State Colleges and Universities Board of Trustees and Leadership Council Joint Meeting. Retrieved from https://www.minnstate.edu/board/minutesdocs/2019/072319\%20Minutes\%20Joint\%20Board\%20and\%20Leadership\%20July\%2023\%2020 19,\%20Final.pdf

Minnesota State Colleges and Universities. (n.d.). Minnesota State Equity 2030. Retrieved from https://www.minnstate.edu/Equity2030/index.html

Popken, B. (2015, August 6). College textbook prices have risen 1,041 percent since 1977. Retrieved from https://www.nbcnews.com/feature/freshman-year/college-textbook-prices-have-risen-812percent-1978-n399926

Senack, E. (2015). Open textbooks: The billion-dollar solution. Student PIRGS. Retrieved from https://studentpirgs.org/2015/02/24/open-textbooks-billion-dollar-solution/

U.S. PIRG. (2014). Report: Make higher education affordable. Retrieved from https://uspirg.org/reports/usp/fixing-broken-textbook-market

Vander Waal Mills, K.E., Gucinski, M., \& Vander Waal, K. (2019). Implementation of open textbooks in community and technical college biology courses: The good, the bad, and the data. CBE-Life Sciences Education, 18(3), 1-11. doi: 10.1187/cbe.19-01-0022 
Wiley, D. (2016). The tidewater z-degree and the INTRO model for sustaining OER adoption. Education Policy Analysis Archives, 24(41), 1-15. doi: 10.14507/epaa.v23.1828

William + Flora Hewlett Foundation. (n.d.). Open Education. Retrieved from https://hewlett.org/strategy/open-education/ 